\title{
Yields of Bronchoscopy and Bronchoalveolar Lavage in Diagnosis of Undefined Persistent Wheezy Chest Among Children
}

\author{
Hussein Mohamed Ibrahim El-Assal ${ }^{1}$, Hatem Refaat Hablas ${ }^{1}$, Mohamed Sami El-Hakim², Mohamed \\ Ibrahim Mohamed Abo Sekkeen ${ }^{1}$
}

\author{
Departments of ${ }^{1}$ Pediatrics and ${ }^{2}$ Pathology, Faculty of Medicine, Al-Azhar University, Cairo, Egypt
}

Corresponding author: Mohamed Ibrahim Mohamed Abo Sekkeen, Mobile: 00201001547870, E-Mail: aabosekken@gmail.com

\begin{abstract}
Background: Wheezing in infants and children is a common problem presented to primary care offices. Approximately 25 to 30 percent of infants will have at least one episode of wheezing. By three years of age, an episode of wheezing will have occurred in 40 percent of children, and by six years of age, almost one half of children will have at least one episode of wheezing. Most infants and children with recurrent wheezing have asthma, but other causes should be considered in the differential diagnosis. The most common diagnoses in children with persistent wheezing are asthma, allergies, gastroesophageal reflux, infections, foreign body aspiration and bronchopulmonary dysplasia. Objective: the present study was designed to evaluate the clinical role of different types of bronchoscope and bronchoalveolar lavage in diagnosis of undefined persistent wheezy chest among the age group from 6 months to 14 years, at Al-Azhar University Hospitals in Cairo. Patients and Methods: This a prospective study was conducted on 50 children complaining from different respiratory problems who had enrolled consecutively from the Outpatient Clinic and the Inpatient Pediatric Department of Al-Azhar Faculty of Medicine University Hospitals (AL-Hussein and Bab-Elshareya), from January 2017 to December 2018. Patients ages ranged between 6 months and 14 years. Results: as regard distribution of patients according to post bronchoscopy diagnosis, the study showed that foreign body inhalation $\mathrm{n} 16$ (32\%), Chronic aspiration n9 (18\%), tracheobronchomalacia n2 (4\%), bronchial mass n1 (2\%), streptococcus pneumonia n7 (14\%), hemophilus influenza n2 (4\%), staphylococcus aureus n3 (6\%) and pseudomonas aeuroginosa n2 (4\%), candida albicans n1 (2\%), Mycobacterium TB n1 (2\%). Conclusion: It could be concluded that if radiography is normal and the child continues to wheeze, bronchoscopy should be the next step, the bronchoscope is a valuable tool in the diagnosis and treatment of airway disorders in children. It has a good safety profile with rarely reported life threatening or long-standing complications.
\end{abstract}

Keywords- Persistent wheezy chest, Bronchoscopy, Bronchoalveolar lavage.

\section{INTRODUCTION}

Wheezing in infants and children is a common problem presented to primary care offices. Approximately 25 to 30 percent of infants will have at least one episode of wheezing. By three years of age, an episode of wheezing will have occurred in 40 percent of children, and by six years of age, almost one half of children will have at least one episode of wheezing. Most infants and children with recurrent wheezing have asthma, but other causes should be considered in the differential diagnosis, Children with continuous wheezing for at least 1 month or minimum three episodes of wheezing in 2 months are defined as persistent wheezy children ${ }^{(1)}$. Chest radiographs is indicated in children who present with unexplained wheezing that is unresponsive to bronchodilators or with recurrent wheezing. Plain- film radiography can identify congenital anomalies of the lung, parenchymal lung disease, and some foreign bodies and cardiac abnormalities, If radiography is normal and the child continues to wheeze, bronchoscopy should be the next step ${ }^{(2)}$. Bronchoscopy is a diagnostic and therapeutic procedure that permits direct visualization of normal and pathological alterations of the upper and lower airways. Expert knowledge of airway anatomy is a prerequisite for successful performance of the procedure. The major advantages of the flexible bronchoscope include the ability to insert it nasally, orally or through a tracheostomy to visualize apical segments of upper lobes as well as segmental and subsegmental bronchi in all lobes ${ }^{(3)}$. The use of the flexible bronchoscope has progressively increased to cover the visual diagnosis of the upper and lower airways lesions as well as interventional, therapeutic and supportive work such as: Bronchoalveolar lavage (BAL) for cytological, virological, bacteriological and immunological 
data; bronchoscopic intubation for anesthetists, catheterization of fistulae; intra operative assistance for cardiac and airway surgery; removal of distal foreign bodies not reached by rigid instruments, and limited selective segmental or lobar bronchographic procedures ${ }^{(4)}$. The role of BAL in monitoring interstitial diseases The most common method used for diagnosing GERD related pulmonary aspiration. The lipid content of the refluxed food is phagocytosed by alveolar macrophages which can be stained with oil red $\mathrm{O}$ stain (1-\{2,5-dimethyl-4-(2-5dimethylphenyl) phenyldiazenyl $\}$ azonapthalen-2-ol). But it does not differentiate aspirated lipid from lipid broken

\section{Patients and Methods:}

This prospective study included a total of 50 children complaining from different respiratory problems attended at the Outpatient Clinic and the Inpatient Pediatric Department of Al-Azhar Faculty of Medicine, University Hospitals (Pulmonology Unit at AL-Hussein and BabElshareya). Approval of the Ethics Board of Al-Azhar University and a written informed consent from all the subjects were obtained. This study was conducted between January 2017 to December 2018.

\section{Inclusion criteria:}

1- Any child suffers from undiagnosed persistent wheezy chest.

2- Age from 6 months to 14 years.

3- Patients with normal coagulation profile.

\section{Exclusion criteria:}

1- Children $<6$ months or $>14$ years old.

2- Children with bronchial asthma or reactive airway disease.

3- Children with diagnosed chest problem (e.g. GERD, BPD, foreign body aspiration, or cystic fibrosis)

4- Children with hematological proplem.

5- Children with other associated extra pulmonary Co-morbid diseases e.g.: liver, cardiac or renal diseases.

The enrolled children were subjected to the following:

A. HISTORY: Full Detailed history especially of pulmonary symptoms and symptoms of other system affection.

B. CLINICAL EXAMINATION:

1. General examination especially for vital signs \& body measurements

2. Systemic review including all body systems:

3. Local Examination of the chest: down from intrinsic alveolar membrane phospholipids causing a false- positive results ${ }^{(5)}$. LLMI was described by Corwin and Irwin to improve specificity by quantifying the lipid content of alveolar macrophages . LLMI above 100 is considered positive for aspiration. The sensitivity was $100 \%$ for pulmonary aspiration, but also the specificity was only $57 \%^{\left({ }^{(6)}\right.}$.

The aim of the current work was to evaluate the clinical role of different types of bronchoscope and bronchoalveolar lavage in diagnosis of undefined persistent wheezy chest among the age group from 6 months to 14 years, at Al-Azhar University Hospitals in Cairo.

a. Inspection: for signs of respiratory distress, the shape of the chest, symmetry and if there is scars, pigmentation or dilated veins.

b. Palpation: of the trachea central or not, if there is local chest tenderness, and tactile vocal fremitus.

c. Percussion: of different areas of the chest.

d. Auscultation: for air entry, breath sound and adventitious sound.

\section{INVESTIGATION:}

1. Routine investigations these include: CBC, CRP, ESR, and liver \& kidney functions, PT, PTT and INR.

2. Plain chest X- Ray and CT chest (if indicated).

3. Pulmonary function test (if patient condition permissible).

4. Tuberculin test (in suspected cases).

5. Broncho-alveolar lavage:

6. Cytology including lipid-laden macrophages index to exclude reflux.

7. Culture and sensitivity.

\section{Patient preparation:}

All the patients were fasted for at least 4-6 hours prior to the procedure. Atropine $0.01 \mathrm{mg} / \mathrm{kg}$ IM was given about 30 minutes before the procedure. The procedure was carried out under strict aseptic precautions by either nasal or oral route. This fasting period is essential to avoid aspirations of gastric content according to the recommendation of American Society of Anesthesiologists.

Psychological preparation was achieved by an age appropriate explanation of the procedure and reassurance to the child and the family. This can reduce preprocedure anxiety, and reduce sedation requirement and improve cooperation ${ }^{(7)}$. 


\section{Technique:}

Both general and local anesthetic techniques are available to experienced practitioners but currently gaseous general anesthetic techniques were used in all centers. This technique has evolved because of a general preference to have an experienced anesthetist present to assist in airway management particularly when difficult airway lesions are present. Anesthetists should also be concerned about the use of "conscious sedation" in children ${ }^{(8)}$.

\section{General Anaesthetic Technique:}

The general anaesthetic technique is usually a gaseous induction \& after four to six hours of fasting. Halothane, sevoflurane and oxygen with intravenous atropine and succinylcholine are the drugs commonly used for young children. However, there are different strengths of lignocaine solutions, sprays and delivery methods vary from the cass needle approach to direct deposition through the bronchoscope or even nebulizer delivered drug. Care must be taken not to exceed the total topical dose of $3-4 \mathrm{mg} / \mathrm{kg}$ for the former modes while doses up to $7-8 \mathrm{mg} / \mathrm{kg}$ for the nebulizer route are reported to be safe, Where BAL procedures are to be carried out for bacteriology, lignocaine is usually not applied directly to the airway because of potential bacteriostatic effects, even though streptococcus pneumoniae appears to be the only organism affected ${ }^{(9)}$.

The usual method of airway maintenance is a shared airway procedure with the bronchoscope passed through a connector and facemask, laryngeal mask or endotracheal tube, Recovery time is very short. Often less than 30 mints observation in a recovery ward is required. Most elective procedures are performed in a day surgery setting with one to two hours post procedure observation of the patient before discharge. Postprocedure pain or discomfort rarely occurs.

The advantage of this approach is the control of the airway, the ability to convert to rigid procedures should be necessary to make detailed assessments of upper airway lesions, and to intervene if necessary with other procedures. These procedures should only be performed if supported by anesthetists experienced and trained in paediatric anaesthesia.

\section{Procedure:}

Karl Storz fibro-optic (FOB) bronchoscope (external diameter $3.5 \mathrm{~mm}$ ) was used for the procedure.

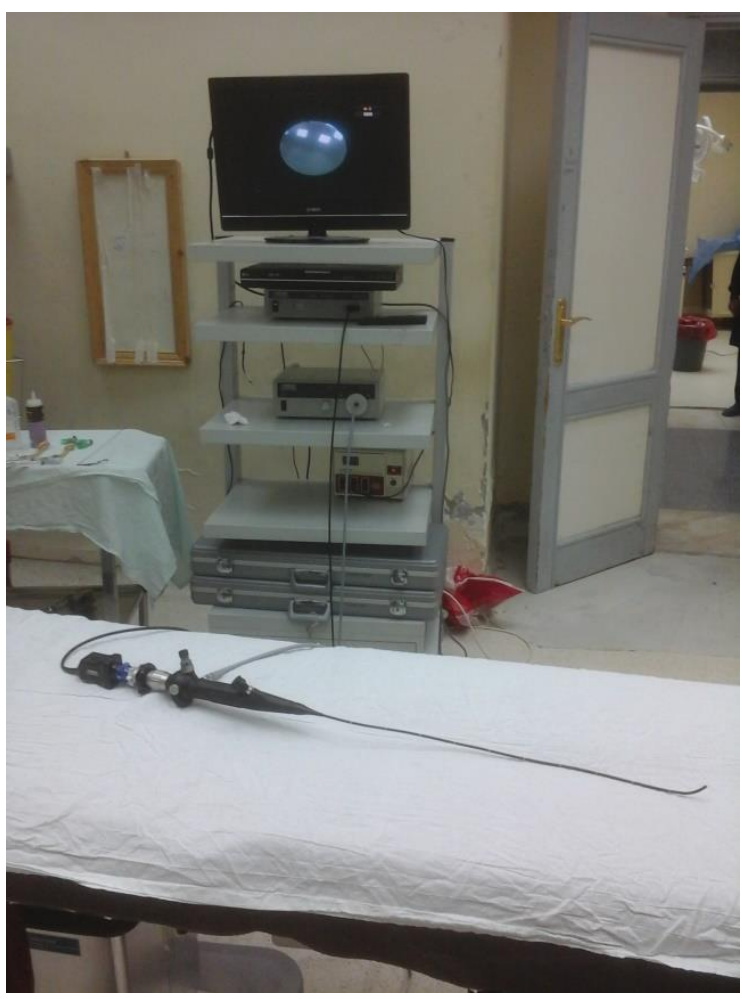

Fig (1) : Work station \& FOB at Al-Azhar University Hospital in Cairo Karl Storz (Germany)

Rigid bronchoscope (with different external diameter) was used when indicated.

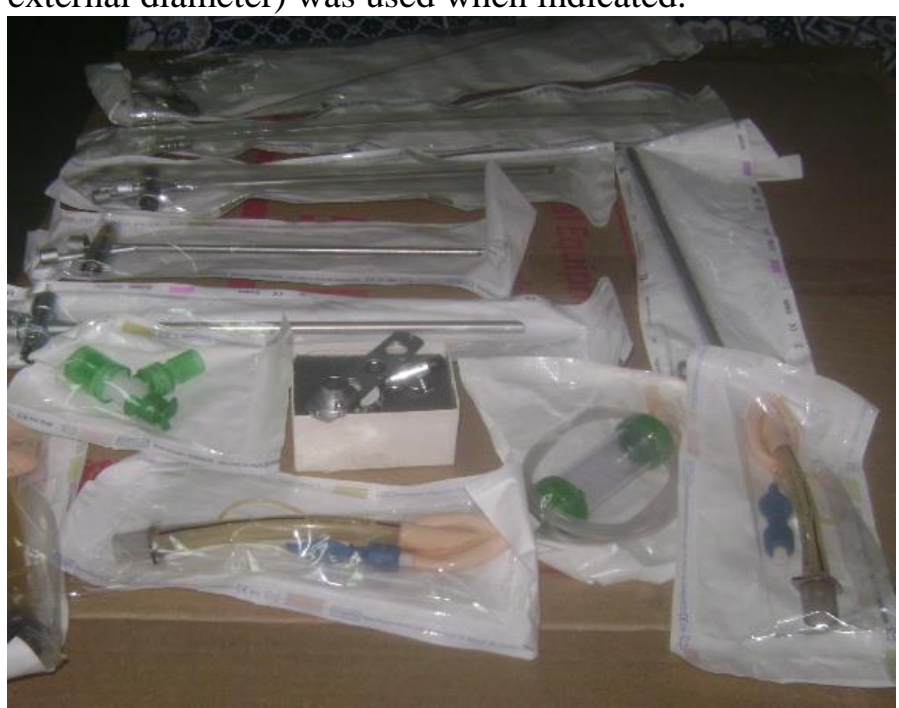

Fig (2): Karl Storz Rigid bronchoscope, laryngeal mask, \& connector at Al-Azhar University Hospitals in Cairo

A quick inspection of the bronchial tree of the normal side was undertaken followed by inspection of the affected side. Bronchoalveolar lavage was done wherever indicated. The bronchoscope was wedged in a segmental bronchus with pathology and bronchoalveolar lavage carried out in three aliquots by injecting normal saline $(2 \mathrm{ml} / \mathrm{kg})$ 
through the working channel. Most bronchoscopy procedures were performed in an operating theater equipped specially for this purpose, under general anaesthesia.

All the monitoring parameters during the procedures were registered in addition to any complications during the anesthesia as well as the procedure itself will be recorded. The child was followed after the procedure till the condition becomes stable, so that any complications or deviation in his clinical condition was interfered and recorded.

The patient was placed on the examination table in the supine position, his head was slightly elevated by using a small firm pillow and neck was slightly flexed forward with a disposable towel to catch any secretion.

Prior to initiating the procedure, the suction channel \& rod lens were checked to ensure proper functioning and the distal $2 \mathrm{~cm}$ of the shaft was lubricated with jelly.

\section{RESULTS}

The results of this study were tabulated in different tables for statistical evaluation of study. 50 Patients were involved in this study $22(44 \%)$ of them were males \& $28(56 \%)$ of them were females, their ages ranged from 6 months to 14 years, The attended
The bronchoscopic handle was held in the left hand and the insertion tube in the right hand. Then the lubricated tip was introduced through the nose, laryngeal mask, face mask or endotracheal tube. The instrument was advanced under direct vision to examine the entire vocal cord, arytenoids, larynx, trachea \& bronchi.

Post-bronchoscopy care:

After completion of the procedure, the patient was transferred to the recovery area until the patient become stable, fully awake and alert. Patients were monitored carefully for any complications and their vital signs were recorded. The mother was instructed not to give any diet until the patient is fully awake and alert ${ }^{(\mathbf{8})}$.

Most our cases passed safely with no complications. Regarding the bronchoscope and its accessories the cleaning and disinfecting procedures were carried out strictly according to the manufacturer's guideline.

patients were complaining from different respiratory problems (chronic cough, persistent wheeze, difficult breathing, \& frequently recurrent croupy cough).

Bronchoscopic procedures were done in the operating theater at Bab- Elshareya University Hospital under general anesthesia.

Table (1): Demographic data of studied patients $(n=50)$.

\begin{tabular}{|c|c|}
\hline Variables & All cases (n= 50) \\
\hline Age (years): & $2.8 \pm 2.2$ \\
Mean \pm SD (range) & $9(18 \%)$ \\
\hline Age groups (\%): & $35(70 \%)$ \\
6 months- 1year & $5(10 \%)$ \\
1-5 years & $1(2 \%)$ \\
5-10 years & $28(56 \%)$ \\
$>10$ years & $22(44 \%)$ \\
\hline Sex: & \\
Females & $37(74 \%)$ \\
Males & $13(26 \%)$ \\
\hline Residence: & \\
Urban & \\
Rural & \\
\hline
\end{tabular}

The table shows that the age between 1-5 years (70\%) was the frequent age of bronchoscopic procedures \& Females (56\%) more than Males (44\%).

Table (2): Comparison of complaints among age groups: 
Hussein El-Assal et al.

\begin{tabular}{|l|c|c|c|c|c|}
\hline \multicolumn{1}{|c|}{ Complaint } & $\begin{array}{c}\mathbf{6 m - 1 y r} \\
(\mathbf{n = 9})\end{array}$ & $\begin{array}{c}>\mathbf{1 - 5} \mathbf{y r s} \\
(\mathbf{n = 3 5})\end{array}$ & $\begin{array}{c}>\mathbf{5 - 1 0} \text { yrs } \\
(\mathbf{n = 5})\end{array}$ & $\begin{array}{c}>\mathbf{1 0} \text { yrs } \\
(\mathbf{n = 1})\end{array}$ & P-Value \\
\hline Chronic cough & $\begin{array}{c}3 \\
(33.33 \%)\end{array}$ & $6(17.14 \%)$ & $2(40 \%)$ & $1(100 \%)$ & $<0.01$ \\
\hline Difficult breathing & $\begin{array}{c}1 \\
(11.11 \%)\end{array}$ & $\begin{array}{c}12 \\
(34.28 \%)\end{array}$ & $0(0 \%)$ & $0(0 \%)$ & $<0.01$ \\
\hline Persistent wheeze & $\begin{array}{c}5 \\
(55.55 \%)\end{array}$ & $\begin{array}{c}15 \\
(42.85 \%)\end{array}$ & $2(40 \%)$ & $0(0 \%)$ & $<0.01$ \\
\hline Recurrent croupy cough & $0(0 \%)$ & $2(5.71 \%)$ & $1(20 \%)$ & $0(0 \%)$ & $<0.01$ \\
\hline
\end{tabular}

p-value >0.05 NS; *p-value <0.05 S; **p-value <0.001 HS

There was highly statistically significant difference between complaints among age groups.

Table (3): Chest Examination of studied patients

\begin{tabular}{|l|c|c|}
\hline \multicolumn{1}{|c|}{ Variable } & Frequency & Percentage (\%) \\
\hline $\begin{array}{l}\text { Inspection: } \\
\text { Normal }\end{array}$ & 14 & $(28 \%)$ \\
Respiratory distress & 17 & $(34 \%)$ \\
$\quad$ Tachypnea & 16 & $(32 \%)$ \\
$\quad$ Chest indrowing & 3 & $(6 \%)$ \\
$\quad$ Cyanosis & & $(54 \%)$ \\
$\quad$ Palpation: & 27 & $(46 \%)$ \\
$\quad$ Palpable rhonchi & 23 & $(58 \%)$ \\
\hline $\begin{array}{l}\text { Auscultation: } \\
\text { Air entry }\end{array}$ & 29 & $(26 \%)$ \\
Equal & & $(16 \%)$ \\
Diminished on right side & 13 & \\
Diminished on left side & 8 & $(58 \%)$ \\
Adventitious sounds & & $(88 \%)$ \\
$\quad$ Crepitations & 29 & $(16 \%)$ \\
\hline Rhonchi & 44 & \\
\hline
\end{tabular}

The table shows most cases of this study have respiratory distress \& equal air entry on both sides with positive auscultatory chest findings.

Table (4): Chest CT findings of the studied patients $(n=50)$

\begin{tabular}{|ll|c|c|}
\hline \multicolumn{1}{|c|}{ Variable } & Frequency & Percentage(\%) \\
\hline Right lung & Normal & 24 & $(48 \%)$ \\
& Consolidation & 24 & $(48 \%)$ \\
& Bronchiectasis & 2 & $(4 \%)$ \\
& & & $(42 \%)$ \\
Left lung & Normal & 21 & $(54 \%)$ \\
& Consolidation & 27 & $(4 \%)$ \\
& Bronchiectasis & 2 & $(90 \%)$ \\
Mediastinum & Normal & & $(10 \%)$ \\
& Hilar Lymphadenopathy & 45 & 5 \\
\hline
\end{tabular}

The table shows most cases in this study had evidence of consolidation collapse \& normal mediastinum.

Table (5): Comparison bronchoscopic findings among age groups: 
Yields of Bronchoscopy and Bronchoalveolar Lavage....

\begin{tabular}{|c|c|c|c|c|c|}
\hline Variable & $\begin{array}{c}6 \mathrm{~m}-1 \mathrm{yr} \\
(\mathrm{n}=9)\end{array}$ & $\begin{array}{c}>1-5 \text { yrs } \\
(n=35)\end{array}$ & $\begin{array}{c}>5-10 y r s \\
(n=5)\end{array}$ & $\begin{array}{c}>10 \text { yrs } \\
(n=1)\end{array}$ & P-Value \\
\hline Normal findings & $2(22.22 \%)$ & $6(17.14 \%)$ & $1(20 \%)$ & $0(0 \%)$ & $<0.001$ \\
\hline Pus from right bronchus & $1(11.11 \%)$ & $0(0 \%)$ & $1(20 \%)$ & $0(0 \%)$ & $>0.05$ \\
\hline Pus from left bronchus & $0(0 \%)$ & $3(8.57 \%)$ & $1(20 \%)$ & $0(0 \%)$ & $<0.05$ \\
\hline Inflamed bronchial mucosa & $3(33.33 \%)$ & $11(31.42 \%)$ & $0(0 \%)$ & $1(100 \%)$ & $<0.001$ \\
\hline Foreign body & $1(11.11 \%)$ & $15(42.85 \%)$ & $0(0 \%)$ & $0(0 \%)$ & $<0.001$ \\
\hline Tracheobronchomalacia & $2(22.22 \%)$ & $0(0 \%)$ & $0(0 \%)$ & $0(0 \%)$ & $>0.05$ \\
\hline Tracheobronchial mass & $0(0 \%)$ & $0(0 \%)$ & $1(20 \%)$ & $0(0 \%)$ & $>0.05$ \\
\hline Inflamed larynx and epiglottis & $0(0 \%)$ & $0(0 \%)$ & $1(20 \%)$ & $0(0 \%)$ & $>0.05$ \\
\hline
\end{tabular}

p-value $>0.05 \mathrm{NS} ; *$ p-value $<0.05 \mathrm{~S} ; * *$ p-value $<0.001 \mathrm{HS}$

Regarding the table findings of bronchoscopy among the 4groups, we found that foreign body inhalation and inflamed bronchial mucosa were more common in group 2, this difference was highly statistically significant $\quad(p$-value $<0.001)$.

Table (6): Relation between CT chest findings and post bronchoscopy diagnosis among patients $(\mathbf{n}=\mathbf{5 0})$.

\begin{tabular}{|c|c|c|c|c|c|}
\hline \multirow{3}{*}{ Bronchoscopic \& BAL findings } & \multicolumn{4}{|c|}{ CT Chest finding } & \multirow[b]{3}{*}{ p-value } \\
\hline & \multicolumn{2}{|c|}{ Positive } & \multicolumn{2}{|c|}{ Negative } & \\
\hline & No. & $\%$ & No. & $\%$ & \\
\hline Pus from bronchus & 6 & $12 \%$ & 2 & $4 \%$ & $<0.001 * *$ \\
\hline Inflamed bronchial mucosa & 15 & $30 \%$ & 9 & $18 \%$ & $0.001 *$ \\
\hline Foreign body inhalation & 16 & $32 \%$ & 14 & $28 \%$ & 0.134 \\
\hline $\begin{array}{l}\text { Elevated lipid laden macrophages as a marker for Chronic } \\
\text { aspiration }\end{array}$ & 3 & $6 \%$ & 1 & $2 \%$ & 0.479 \\
\hline Tracheobronchomalacia & 2 & $4 \%$ & 4 & $8 \%$ & 0.059 \\
\hline Inflamed larynx and epiglottis & 1 & $2 \%$ & 0 & $0 \%$ & $<0.001 * *$ \\
\hline Tracheobronchial mass & 1 & $0 \%$ & 0 & $0 \%$ & $<0.001 * *$ \\
\hline
\end{tabular}

p-value $>0.05 \mathrm{NS} ;{ }^{*}$-value $<0.05 \mathrm{~S} ;{ }^{* *}$-value $<0.001 \mathrm{HS}$

This table showed statistically significant relation between CT findings and Bronchoscopic \&BAL findings

\section{DISCUSSION}

The flexible bronchoscope has become widely used by pediatric pulmonologists as a diagnostic and therapeutic tool.

The fifty patients who enrolled into this study and underwent flexible bronchoscopy, 22 $(44 \%)$ of them were males \& $28(56 \%)$ of them were females. Their age ranged from 1 to 8 years, median [IQR] age was (2.8 years $\underline{\mathbf{2} .2}$ years).

As the unit has only one size of the flexible fibreoptic bronchoscope for the time being (Karl Storz Germany, size 3.6mm), the age of the enrolled patients limited between 6 months - 14 years, as there is difficult passage of the size available in children less than 6 months of age \& small image in children more than 14 years of age.
The patients attended the pulmonology unit from different governorates in Egypt, 37 patients $(74 \%)$ came from urban areas \& 13 patients $(26 \%)$ came from rural areas. This difference may be explained on the basis that the bronchoscopy procedure for children is not available in many areas. Although the bronchoscopy unit is starting, the patients referred from different locations, this might be explained by the good reputation of the pediatric pulmonology unit, Al-Azhar University, and the distinguished services offered to the pediatric patients, as well as the service is free of charge till now.

The main presenting pulmonary manifestations in this study were as follow: 16 patients $(32 \%)$ had persistent wheeze, 14 
patients (28\%) had chronic cough, 13 patients (26\%) had difficult breathing, 7 patients (14\%) had history of recurrent croup.

Wood et al ${ }^{(\mathbf{8})}$, their study was done on 192 patients age ranged from 1 month to 16 years and showed the most common complaint indicated for pediatric bronchoscope were as follow: (persistent stridor, unresolving or recurrent pneumonia, undefined persistent wheeze, suspected foreign body, chronic cough, unknown etiology of radiological abnormalities $\&$ hemoptysis) respectively.

Explanation of the difference from our study is that Wood $\boldsymbol{e t}$ al. study ${ }^{\left({ }^{(8)}\right.}$, was done from the neonatal period as laryngeomalacia is a common cause of stridor which usually improved during $1^{\text {st }} 6$ months of life.

Another study done by Priftis $\boldsymbol{e t ~ a l . ~}{ }^{\left({ }^{(10)}\right.}$, it was done on 92 patients from 12 months to 5 years and show the following complaints indicated for bronchoscopy: persistent wheeze, chronic cough, persistent stridor common indications of bronchoscope respectively. This study agrees with our study as persistent pneumonia, chronic cough \& foreign body inhalation the most common indications for pediatric bronchoscope.

In this study 50 patients had bronchoscopic procedures performed at operating theater of Bab- Elshareya University hospital.

In this study, 34 patients (68\%) used flexible bronchoscope only, and 16 patients (32\%) used both bronchoscopes (the flexible \& the rigid).

All bronchoscopic procedures (100\%) were done under general anesthesia (deep sedation) using sevoflurane by inhalation followed by intravenous propofol. The following medications were used during the procedures: normal saline in $82 \%$ of the procedures, lidocaine in $4 \%$ albuterol in $6 \%$, epinephrine in $6 \%$, dexamethasone in $78 \%$, hydrocortisone in $12 \%$, cyclocapron $2 \%$ and succinylcholine in $48 \%$.

Somu et al. (11), done on 582 pediatric patients, they did the procedure under local anesthesia only, with no complications reported.

Jaggar et al. ${ }^{(12)}$, their study was done using conscious sedation.

The technique of sedation used depends on many factors: respiratory status, psychological and emotional status of the patient, underlying disease, drugs available, availability of an anesthetists and type(s) of procedure(s) to be performed.
In our study mean time of bronchoscopic procedures $(16 \pm 4)$ minutes and recovery time usually take about 30 minutes.

National Heart, Lung and blood institute (NHLBI) ${ }^{(13)}$ report that: the bronchoscopy itself usually lasts about 30 minutes. But the entire procedures including preparation and recovery time, takes about 4 hours.

Although the population enrolled in this study may appear heterogeneous in terms of age, indications, or underlying disease, it reflects clinical practice in a Pediatric respiratory unit. There are no strict definitions of complications during FB. Thus the criteria selected, especially the cut-off value of $90 \%$ $\mathrm{Sa}, \mathrm{O} 2$ may appear arbitrary, and brief periods of desaturation to just $<90 \% \mathrm{Sa}, \mathrm{O} 2$ may be considered to constitute a minor complication. The present results show that FB is well tolerated in most cases and that the risk of major complications is low. Minor complications were observed in 4 patients $(8 \%)$ of cases and no major complications occurred. None of the children died during the procedure, and no respiratory deterioration due to bronchoscopy that required intensive care was observed.

In our study chest x-ray were done for all cases and show the followings: the right lung 20 patients (40\%) had no abnormal findings, 14 patients $(28 \%)$ had hyperinflation, 4 patients (8\%) had persistent collapse and 13 patients (26\%) had infiltration. The left lung 23 patients (46\%) had no abnormal findings, 13 patients $(26 \%)$ had hyperinflation, 4 patients $(8 \%)$ had persistent collapse and 11 patients $(22 \%)$ had infiltration. The mediastinum 46 patients (92\%) had normal Mediastinum, 3 patients $(6 \%)$ had mediastinum shifted to the right and 1 patient (2\%) had mediastinum shifted to the left.

In our study chest CT were done for all cases and show the followings: the right and left lungs 21 patients (42\%) had normal right \&left lung, 27 patients (54\%) consolidation collapse and 2 patients (4\%) had bronchiectatic changes. The mediastinum 45 patients $(90 \%)$ had normal Mediastinum \& 5 patients (10\%) had hilar Lymphadenopathy. In our study the main bronchoscopic findings were as follow: 16 patients (32\%) had foreign body impaction, 15 patients $(30 \%)$ had inflamed mucosa of major airway, 9 patients $(18 \%)$ had normal bronchoscopic findings, 2 patients (4\%) had pus coming from right bronchus, 4 patients (8\%) had pus coming from left bronchus, 1 patients $(2 \%)$ had tracheobronchial masses, 3 
patients (6\%) had segmental tracheobronchomalacia and 1 patient $(2 \%)$ had inflamed larynx and epiglottis..

Several studies were done and show different bronchoscopic findings the most one agree with our study which done by Wood $\boldsymbol{e t}$ al. ${ }^{(8)}$, University of Cincinnati College of Medicine) which show the most common findings of bronchoscope was extraction of foreign body followed by inflammation of the airway due to recurrent infections.

In our study Bronchoalveolarlavage was done for 34 patients (68\%), we found that the yield of BAL culture was $(47.06 \%)$. Positive cultures were distributed evenly between patients despite the fact that most of them received antibiotics prior to bronchoscopy. This high yield of cultures compared to those reported by other published studies may be explained by the nature of our patient mix. Contamination of samples is another major issue that may affect laboratory outcomes, even when using the most standardized techniques. As with any other test, there is a risk of false positive/false negative results in BAL cultures currently, however, these are the most accurate tools at our disposal. Nevertheless, clinicians should consider the general condition of the patient and other laboratory tests to obtain an integrated view of the accuracy of any given results. The result of BAL cytology as follows: 15 samples (44.11\%) had normal cytology, 10 samples $(29.41 \%)$ had increase neutrophil \& 4 samples $(11.76 \%)$ had increase lymphocyte, 12 samples $(35.29 \%)$ had increase macrophages, and 9 samples $(26.47 \%)$ had LLMI $>100$.

The results of BAL culture as follow: 18 samples $(52.94 \%)$ had no growth, 7 samples $(20.58 \%)$ had streptococcus pneumoniae, 3 samples (18.82\%) had staphylococcus aureus, 2 samples (5.88\%) had hemophilus influenzae, 2 samples $(5.88 \%)$ had pseudomonas aeruginosa, 1 sample $(2.94 \%)$ had Candida albicans \& 1 sample (2.94\%) had mycobacterium TB.

There are many other analytic assays for BAL such as immunological, chemical \& others but not performed at our units. BAL has become a valuable procedure because, unlike lung biopsy is less invasive, well tolerated by patients, insurance and low percentage of complications, even in infants.

\section{-There were several studies done at different centers:}

Study were done by Gidaris et al. ${ }^{(14)}$, on 50 child, age ranged from one month to 16 years and show the followings on BAL microbiology: 10 child (20\%) have no growth, 8 child (16\%) have streptococcus pneumoniae, 6 child (12\%) have pseudomonas, 5 child $(10 \%)$ have Candida albicans, 5 child (10\%) have staph aureus \& 4 child (8\%) have kelebsilla.

Other study were done at Department of Respiratory Medicine, Children's University hospital, Temple St, Dublin from period (2005 to 2010) on 180 patients age ranged from 2 months to 17 years and showing that: 124 patients (69\%) have no growth, 22 patients (12\%) have streptococcus pneumoniae, 10 patients (18\%) have hemophilus influenza, 9 patients $(5 \%)$ have staph aureus \& 4 patients (2\%) have moraxella catarallis.

Both studies agree with our study as most patients have no evidence of growth of organisms and streptococcus pneumonia most common organism isolated from BAL.

Other study was done by Terkawi et al. ${ }^{(15)}$. on 149 patients and show the common organisms isolated from BAL as follow: 21 patients $(39.6 \%)$ have Pseudomonas aeruginosa , 6 patients $(11.3 \%)$ have Acinetobacter baumannii , 4 patients (7.5\%) have Escherichia coli, 4 (7.5\%), Enterobacter cloacae 4 (7.5\%), Streptococcus pneumoniae 3 patients $(5.7 \%)$ have Staphylococcus aureus, 3 patients $(5.7 \%)$ have Haemophilus influenzae \& 3 patients (5.7\%) have Stenotrophomonas maltophilia. This study disagrees with our study as the commonest organism isolated from BAL was Pseudomonas aeruginosa.

Bronchoscopy, like all invasive procedures, has its pros and cons. It carries the risk of complications from the inserted bronchoscope, extensive lavage, and anesthetic medications. In our study $8 \%$ of patients developed nonlifethreatening complications. The most common was transient desaturation. A good understanding of procedural complications can better prepare for appropriate patient management as well as aid in counseling parents. Consistent with our findings, desaturation was the most common reported complication. Previous authors have used widely variable reductions in $\mathrm{SpO} 2$ to define desaturation leading to significant heterogeneity. 
For instance: Nussbaum et al. ${ }^{(16)}$, defined desaturation as a drop in SpO2to 65\% $80 \%$. Rodri'-guez et al. ${ }^{(17)}$, defined it as a drop in SpO2of $10 \%$ or more below the baseline, while still other studies did not define the term at all. It is important to realize that desaturation in children is particularly dangerous and can rapidly cause bradycardia and even cardiac arrest. Therefore, prompt response and precise coordination and cooperation between the pulmonologist and anesthesiologist are essential. In our study 46 patients $(92 \%)$ had no evidence of complications, 4 patients $(8 \%)$ had desaturation.

\section{Several studies were done on pediatric bronchoscope and show different numbers and types of complications:}

Schnapf et al. ${ }^{(18)}$, Oxygen desaturation has been reported. to be more frequent complications in younger infants. Falls in oxygen saturation are common particularly whilst the bronchoscope is in the mid trachea and can occur despite oxygen supplementation; however, there is a decreased incidence and severity of hypoxia if oxygen is administered during procedures undertaken using sedation

Picard et al. ${ }^{(19)}$, other reported complications during the procedure are rare, in general occurring in $5 \%$ of procedures. Minor complications include epistaxis, airway bleeding, excessive cough, and transient laryngospasm. Major complications include apnea, bradycardia and Post procedure atelectasis is another possible complication. Few paediatric fatalities have been reported. Spread of infections seems to be a very rare complication.

Manna et al. (20), study done on 148 patients and show: (10.8\%) have desaturation \& (17\%) have hypotension.

Righini et al. ${ }^{(21)}$, study done on 82 patients and show: (2.4\%) have laryngeal spasm \& $(2.4 \%)$ have subglottic edema.

Tang et al. (22), study done on 53 patients and show: (20.8\%) have desaturation, (3.8\%) have mild bleeding \& $(1.9 \%)$ have laryngeal spasm.
Malherbe et al. ${ }^{(23)}$, study done on 52 patients and show: (19\%) have desaturation, $(27 \%)$ have cough \& $(21 \%)$ have apnea.

Peng et al. ${ }^{(24)}$, study done on 725 patients and show $(0.8 \%)$ have laryngeal spasm.

Terkawi et al. ${ }^{(15)}$, study done on 149 patients and show; $(21.5 \%)$ have desaturation, $(0.7 \%)$ have mild bleeding, $(0.7 \%)$ have laryngeal spasm, $(0.7 \%)$ have apnea \& $(0.7 \%)$ have vomiting. most of these study agrees with our study as the most common complications of pediatric bronchoscope was desaturation.

Our study has minimal complications due small numbers of patients involved and major bronchoscopic interventional procedures such as stent, debulking not done and our study not done on neonates or intensive care units.

finally, Bronchoscopy is unethical in a clinical context unless performed with the maximum attention to safety, and in a context in which the potential benefit to the child justifies the perceived risk of the examination. Fully informed consent, in accord with local and national guidelines, is mandatory.

\section{CONCLUSION}

It could be concluded that if radiography is normal and the child continues to wheeze, bronchoscopy should be the next step, the bronchoscope is a valuable tool in the diagnosis and treatment of airway disorders in children. It has a good safety profile with rarely reported life threatening or long-standing complications.

\section{RECOMMENDATIONS}

1. Persistent wheezy children who fail to respond to inhaled bronchodilator and ICS should be thoroughly investigated.

2 . The most commonly aspirated foreign bodies are nuts and seeds. Therefore, it is necessary to instruct parents and child careers not to give any nuts or small seeds to pre-school children. Sure enough, prevention invariably serves better than treatment, even the best one.

3. The aspiration of a foreign body should be suspected in each and every case of a pulmonary infection marked by ambiguous symptoms, especially with concomitant 
atelectasis or emphysema of some parts of pulmonary parenchyma.

4.In the event of a suspected aspiration, even if the preliminary examination on X-ray does not indicate any changes, bronchoscopy should be performed, since it constitutes the best diagnostic and therapeutic method under these circumstances.

5.GERD should be suspected in wheezy children with persistent wheeze even in absence of typical GERD symptoms.

6.For better assessment of GERD associated children wheeze LLMI should be performed.

7.It is recommended to find better instruments not smaller, but with better optics. Better training to ensure skilled performance of clinically needed procedures. Better understanding on the part of pediatrics practitioners of the role of bronchoscope in the diagnosis and management of paediatric patients to develop more appropriate referral patterns. Better practice models to improve the multidisciplinary management of difficult diagnostic and therapeutic dilemmas. Better research applications to improve our understanding of disease and the development of new therapies

\section{REFERENCES}

1. Martinez FD, Wright AL,Taussing LM, Holberg CJ, Halonen M, Morgan WJ(2009): Asthma and wheezing in the first six years of life. The Group Health Medical Associates. N Engl J Med.,332(3):133-138.

2. Bush A(2017): Diagnosis of asthma in children under five. Prim Care Respir J. , 16(1):7-15.

3. Kumaran R, Sung A And Ernst A(2014):Introduction bronchoscope. assets.cambridge.org/97805217/6628 9/frontmatter/9780521766289_frontm atter.pdf

4. Wood $\mathbf{R E ( 2 0 1 2 ) : ~ T h e ~ e m e r g i n g ~ r o l e ~}$ of flexible bronchoscopy in pediatrics clinics. Chest Medicine, 22 (2) 3117.

5. Armstrong DS, Grimwoo d K, CarlinJB, Carzino R, Olinsky A, Phelan PD (2015):BronchoalveolarLavage or Oropharyngeal Cultures to Identify Lower Respiratory Pathogens in Infants With Cystic Fibrosis. Pediatr
Pulmonol., 21:267-275.

6. Farrell S, McMaster C, Gibson D, Shields MD, McCallion WA(2008):

Bronchoalveolar lavage fluid: a specific and sensitive method for diagnosis of GERD-related pulmonary aspiration .J Pediatr. Surg .,41(2):28993.

7. American Society of Anesthesiology (2012): Preoperative fasting and the use of pharmacologic agents to reduce the risk of pulmonary aspiration. Anesthesiology, 90:896-905.

8. Wood RE (2016): The emerging role of flexible bronchoscopy in pediatrics clinics. Chest Medicine, 22 (2): 3117.

9. Olsen KM, Peddicord TE, Campbell D, RuppME (2007). Antimicrobial effects of lidocaine in bronchoalveolar lavage fluid. Antimicrob Chemother., 45:217-219.

10. Priftis KS, Anthracopoulos MB, Mermiri D, Papadopulou A, Xepapadaki P, Tsakanika C et al. (2006). Bronchial hyperresponsiveness, atopy and bronchoalveolar lavage eosinophils in persistent middle lobe syndrome. Pediatr Pulmonol., 41:805-11.

11. Somu , Canny GJ, Levison H(1995): Wheezing in infants and young children.

In: Tinkelman DG, Naspitz CK. Childhood Asthma: pathophysiology and treatment.

$2^{\text {nd }}$ ed. New York, NY: Dekker.

12. Jaggar SI, Haxby E (2004): Sedation, anaesthesia and monitoring for bronchoscopy. Paediatr Respir Rev .,3:321-327

13. National Heart, Lung, and Blood Institute, National Asthma Education and Prevention Program(2012): Guidelines for the diagnosis and management of asthma. https://www.nhlbi.nih.gov/health.../gui delines-for-diagnosis-management-ofasthma

14. Gidaris D, Kanakouli T, Tsakalidou D, Papakostra V, Tzimouli A, Taparkou M (2010): Ventouri Tsanakas Bronchoalveolar lavage in children with inflammatory and non inflammatory lung disease 
Hippokratia , 4:3259

15. Terkawi R, Altirkawi K, Terkawi A, Mukhtar G, Al-Shamrani A (2015):

Flexible bronchoscopy in children: Utility and complications International Journal of Pediatrics and Adolescent Medicine,3:18-27,

16. Nussbaum E (2002): Pediatric fiberoptic bronchoscopy: clinical experience with 2,836 bronchoscopies. Pediatr Crit Care Med .,3: 171-6.

17. Rodri'guez Martı'nez C, Sossa MP(2003): Factors associated with complications caused by bronchoscopy in pediatric patients. Arch Bronconeumol.,39:501e6

18. Schnapf B (2002):Oxygen desaturation during FOB in pediatric patients. Chest, 3: 591-594.

19. Picard E, Schwartz S, Goldemberg S, Glick T, Villa Y, Kerem E (2005): A prospective study of fever and bacteremia after flexible fiberoptic bronchoscopy in children. Chest, 117:573-577.

20. Manna SS, Durward A, Moganasundram S, Tibby SM, Murdoch IA (2006): Retrospective evaluation of a paediatric intensivist- led flexible bronchoscopy service. Intensive Care Med .,32:2026-33.

21. Righini CA, Morel N, Karkas A, Reyt E, Ferretti K, Pin I, Schmerber $\mathbf{S}$ (2009): What is the diagnostic value of flexible bronchoscopy in the initial investigation of children with suspected foreign body aspiration? Int J Pediatr Otorhinolaryngol., 71:138390.

22. Tang LF, Chen ZM (2009): Fiberoptic bronchoscopy in neonatal and pediatric intensive care units: a 5year experience. Med Princ Pract ., 18:305-9

23. Malherbe S, Whyte S, Singh $P$, Amari E, King A, Mark Ansermino J(2010): Total intravenous anesthesia and spontaneous respiration for airway endoscopy in children prospective evaluation. Paediatr Anaesth .,20:4348.

24. Peng Y, Soong W, Lee Y, Tsao P, Yang C, Jeng $M$ (2011): Flexible bronchoscopy as a valuable diagnostic and therapeutic tool in pediatric intensive care patients: a report on 5 years of experience. Pediatr Pulmonol $., 46: 1031-7$ 